\title{
Two-Loop Sudakov Form Factor in a Theory with a Mass Gap
}

\author{
Bernd Feucht, ${ }^{1}$ Johann H. Kühn, ${ }^{1,2}$ Alexander A. Penin, ${ }^{1,3}$ and Vladimir A. Smirnov ${ }^{4,5}$ \\ ${ }^{1}$ Institut für Theoretische Teilchenphysik, Universität Karlsruhe, 76128 Karlsruhe, Germany \\ ${ }^{2}$ Kavli Institute for Theoretical Physics, University of California, Santa Barbara, California 93106, USA \\ ${ }^{3}$ Institute for Nuclear Research, Russian Academy of Sciences, 117312 Moscow, Russia \\ ${ }^{4}$ Institute for Nuclear Physics, Moscow State University, 119992 Moscow, Russia \\ ${ }^{5}$ II. Institut für Theoretische Physik, Universität Hamburg, 22761 Hamburg, Germany
}

(Received 8 April 2004; published 3 September 2004)

\begin{abstract}
The two-loop Sudakov form factor is computed in a U(1) model with a massive gauge boson and a $\mathrm{U}(1) \times \mathrm{U}(1)$ model with mass gap. We analyze the result in the context of hard and infrared evolution equations and establish a matching procedure which relates the theories with and without mass gap, setting the stage for the complete calculation of the dominant two-loop corrections to electroweak processes at high energy.
\end{abstract}

DOI: 10.1103/PhysRevLett.93.101802

Recently a new wave of interest in the Sudakov asymptotic regime $[1,2]$ has risen in connection with higherorder corrections to electroweak processes at high energies [3-10]. Experimental and theoretical studies of electroweak interactions have traditionally explored the range from very low energies, e.g., through parity violation in atoms, up to energies comparable to the masses of the $W$ and $Z$ bosons, e.g., at the LEP or the Tevatron. The advent of multi-TeV colliders like the LHC during the present decade or a future linear electron-positron collider will give access to a completely new energy domain. Once the characteristic energies $\sqrt{s}$ are far larger than the masses of the $W$ and $Z$ bosons, $M_{W, Z}$, exclusive reactions like electron-positron (or quark-antiquark) annihilation into a pair of fermions or gauge bosons will receive virtual corrections enhanced by powers of the large electroweak logarithm $\ln \left(s / M_{W, Z}^{2}\right)$.

The calculation of the two-loop electroweak corrections is an extremely challenging theoretical problem. It is complicated, in particular, by the presence of the mass gap and mixing in the gauge sector. However, the logarithmic corrections are quite insensitive to fine details of the spontaneous symmetry breaking. The calculation of the leading logarithmic (LL) electroweak corrections can be performed using the fields of the unbroken symmetry phase and how the infrared singular virtual photon contribution can be separated within the infrared evolution equation approach [5]. This scheme has been extended to the next-to-leading (NLL) and next-to-next-to-leading logarithmic $\left(\mathrm{N}^{2} \mathrm{LL}\right)$ approximation in Refs. [6,9].

In the study of Sudakov corrections the analysis of the form factor plays a special role since it is the simplest quantity that includes the complete information about the universal collinear logarithms [11] directly applicable to a process with an arbitrary number of fermions. In this Letter we formulate a general matching procedure that relates the logarithmic corrections in the theories with and without mass gap by combining the hard and infrared evolution equation approach with the explicit two-loop
PACS numbers: 12.15.Lk, 12.38.Bx, 12.38.Cy, 13.40.Ks

results for the form factor in an Abelian gauge model. This reduces the calculation of the dominant two-loop corrections to electroweak processes at high energy to a single-mass problem without mixing.

The structure of the Letter is as follows. First, we present the explicit two-loop results for the form factor in a U(1) model with a massive gauge boson. Then we introduce the evolution equations, compute the two-loop corrections to the form factor in a $\mathrm{U}(1) \times \mathrm{U}(1)$ model with mass gap, and establish the matching procedure. Finally, we outline how the approach can be applied to the calculation of the two-loop electroweak corrections to neutral current four-fermion processes.

The vector form factor $\mathcal{F}$ determines the fermion scattering amplitude in an external Abelian field. It is a function of the Euclidean momentum transfer $Q^{2}=$ $-\left(p_{1}-p_{2}\right)^{2}$ where $p_{1,2}$ is the incoming and outgoing fermion momentum and we consider on-shell massless fermions, $p_{1}^{2}=p_{2}^{2}=0$. Let us write the perturbative expansion for the form factor as $\mathcal{F}_{\alpha}(M, Q)=$ $\sum_{n}\left(\frac{\alpha}{4 \pi}\right)^{n} f^{(n)} \mathcal{F}_{B}$ where $\mathcal{F}_{B}$ corresponds to the Born approximation, $f^{(0)}=1$. For the $\mathrm{U}(1)$ model with a gauge boson of mass $M$ in the Sudakov limit $M / Q \rightarrow 0$ the oneloop correction is well known [12]

$$
f^{(1)}=-\mathcal{L}^{2}+3 \mathcal{L}-\frac{7}{2}-\frac{2}{3} \pi^{2},
$$

where $\mathcal{L}=\ln \left(Q^{2} / M^{2}\right)$ and all the power-suppressed terms are neglected. For the two-loop term we find

$$
\begin{aligned}
f^{(2)}= & \frac{1}{2} \mathcal{L}^{4}-3 \mathcal{L}^{3}+\left(8+\frac{2}{3} \pi^{2}\right) \mathcal{L}^{2}-\left[9+4 \pi^{2}\right. \\
& \left.-24 \zeta_{3}\right] \mathcal{L}+\frac{25}{2}+\frac{52}{3} \pi^{2}+80 \zeta_{3}-\frac{52}{15} \pi^{4} \\
& -\frac{32}{3} \pi^{2} \ln ^{2} 2+\frac{32}{3} \ln ^{4} 2+256 \operatorname{Li}_{4}\left(\frac{1}{2}\right),
\end{aligned}
$$

where $\zeta_{3}=1.202057 \ldots$ and $\operatorname{Li}_{4}\left(\frac{1}{2}\right)=0.517479 \ldots$ are the values of the Riemann's $\zeta$ function and the polylogarithm, respectively. In Eq. (2) we do not include the 
contribution due to the virtual fermion loop computed in [10]. For the calculation of the leading power behavior of the two-loop on-shell vertex diagrams with two massive propagators in the Sudakov limit we used the expansion by regions approach [13] (for the application to the Sudakov form factor see also [6]). The method is based on the factorization of the contributions of the dynamical modes characteristic for the Sudakov limit [14] in dimensional regularization. Our result for the contribution of the hard modes agrees with the dimensionally regularized massless result of Ref. [15].

In Fig. 1 the numerical results for the two-loop correction to the form factor in the different logarithmic approximations are plotted as functions of the momentum for the values of $M$ and $\alpha$ typical for electroweak interactions. The two-loop logarithmic terms have a signalternating structure resulting in significant cancellations. In the region of a few $\mathrm{TeV}$ the form factor does not reach the double-logarithmic asymptotics. The quartic, cubic, and quadratic logarithms are comparable in magnitude and dominate the two-loop corrections. Then the logarithmic expansion starts to converge and, after including the linear-logarithmic contribution, provides a very accurate approximation of the total two-loop correction. Such a behavior is typical for the Sudakov limit and holds for the non-Abelian corrections as well $[9,10]$. Note that by rescaling $M \rightarrow e^{3 / 4} M$ in the argument of the logarithm the NLL contribution can be made to disappear. That improves the convergence of the logarithmic expansion and prevents the strong cancellation between the logarithmic terms (see Fig. 2). Still, the $\mathrm{N}^{3} \mathrm{LL}$ contribution is a must for the quantitative approximation.

The asymptotic dependence of the form factor on $Q$ is governed by the linear hard evolution equation [16]. As a consequence, the logarithmic corrections exponentiate.

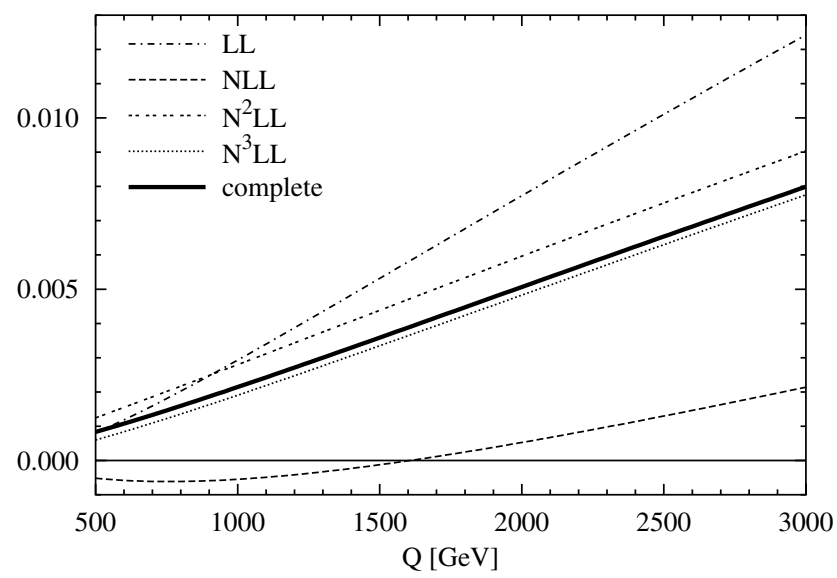

FIG. 1. The two-loop correction to the form factor $\mathcal{F}_{\alpha}(M, Q)$ in LL (including $\alpha^{2} \mathcal{L}^{4}$ ), NLL (including $\alpha^{2} \mathcal{L}^{3}$ ), $\mathrm{N}^{2} \mathrm{LL}$ (including $\alpha^{2} \mathcal{L}^{2}$ ), and $\mathrm{N}^{3} \mathrm{LL}$ (including $\alpha^{2} \mathcal{L}^{1}$ ) approximations and the complete two-loop correction as functions of the momentum transfer for $M=80 \mathrm{GeV}, \alpha /(4 \pi)=3 \times 10^{-3}$.
For the purely Abelian contribution the exponent has a particularly simple form

$$
\begin{aligned}
\mathcal{F}_{\alpha}(M, Q)= & \exp \left(\frac { \alpha } { 4 \pi } \left\{-\mathcal{L}^{2}+\left[3-\frac{\alpha}{4 \pi}\left[-\frac{3}{2}+2 \pi^{2}\right.\right.\right.\right. \\
& \left.\left.\left.\left.-24 \zeta_{3}\right]+\mathcal{O}\left(\alpha^{2}\right)\right] \mathcal{L}\right\}\right) \mathcal{F}_{\alpha}(M, M) .
\end{aligned}
$$

The double-logarithmic term in the exponent is protected against the Abelian multiloop corrections by the properties of the light-cone Wilson loop [17]. Our two-loop result determines the next-to-next-to-next-to-next-toleading logarithmic $\left(\mathrm{N}^{4} \mathrm{LL}\right)$ approximation of the form factor which includes the $\alpha^{n} \mathcal{L}^{m}$ corrections with $m=$ $2 n-4, \ldots, 2 n$ to all orders in $\alpha$.

Let us now turn to the second example with two Abelian gauge bosons of the masses $\lambda$ and $M, \lambda \ll M$, and couplings $\alpha^{\prime}$ and $\alpha$, respectively. We can introduce the infrared evolution equation which governs the dependence of the form factor $\mathcal{F}(\lambda, M, Q)$ on $\lambda$ [5]. The virtual corrections become divergent in the limit $\lambda \rightarrow 0$. According to the Kinoshita-Lee-Nauenberg theorem, these divergences are canceled against the ones of the corrections due to the emission of real light gauge bosons of vanishing energy and/or collinear to one of the onshell fermion lines. The singular behavior of the form factor must be the same in the full $\mathrm{U}_{\alpha^{\prime}}(1) \times \mathrm{U}_{\alpha}(1)$ theory and the effective $\mathrm{U}_{\alpha^{\prime}}(1)$ model with only the light gauge boson. Thus for $\lambda \ll M \ll Q$ the solution of the infrared evolution equation is given by the exponent of Eq. (3) with $M, \alpha$ replaced by $\lambda, \alpha^{\prime}$, and the form factor can be written in a factorized form

$$
\mathcal{F}(\lambda, M, Q)=\tilde{F}(M, Q) \mathcal{F}_{\alpha^{\prime}}(\lambda, Q)+\mathcal{O}(\lambda / M),
$$

where the function $\tilde{F}(M, Q)$ depends on $\alpha$ and $\alpha^{\prime}$ and incorporates all the logarithms of the form $\ln \left(Q^{2} / M^{2}\right)$. It can be obtained directly by calculating the ratio

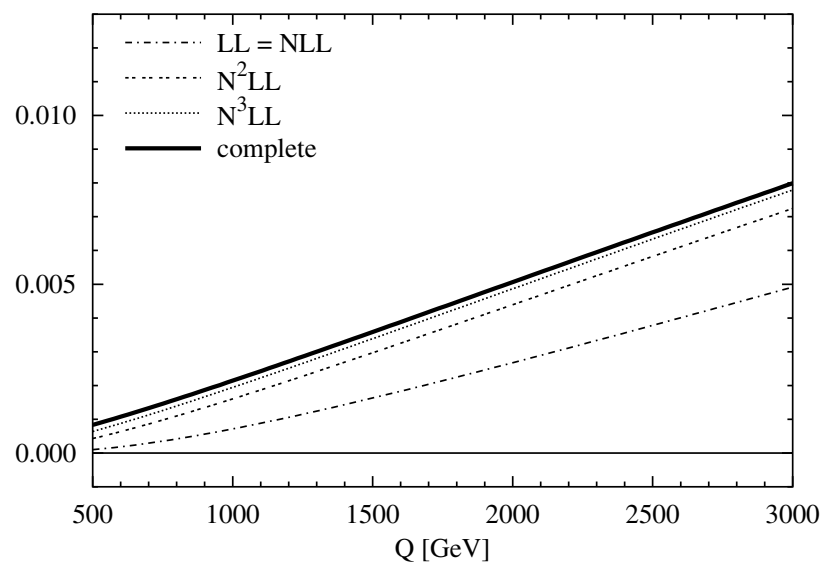

FIG. 2. The same as Fig. 1 after changing the argument of the logarithm. 


$$
\tilde{F}(M, Q)=\left[\frac{\mathcal{F}(\lambda, M, Q)}{\mathcal{F}_{\alpha^{\prime}}(\lambda, Q)}\right]_{\lambda \rightarrow 0} .
$$

Since the function $\tilde{F}(M, Q)$ does not depend on the infrared regularization, we compute the ratio in Eq. (5) with $\lambda=0$ using dimensional regularization for the infrared divergences. The method of calculation of the two-loop diagrams with both massive and massless gauge bosons is similar to the purely massive case. We obtain the twoparameter perturbative expansion $\tilde{F}(M, Q)=$ $\sum_{n, m} \frac{\alpha^{\prime n} \alpha^{m}}{(4 \pi)^{n+m}} \tilde{f}^{(n, m)}$, where $\tilde{f}^{(0,0)}=1, \tilde{f}^{(n, 0)}=0, \tilde{f}^{(0, m)}=$ $f^{(m)}$, and the two-loop interference term reads

$$
\tilde{f}^{(1,1)}=\left(3-4 \pi^{2}+48 \zeta_{3}\right) \mathcal{L}-2+\frac{20}{3} \pi^{2}-84 \zeta_{3}+\frac{7}{45} \pi^{4} .
$$

The numerical structure of the corrections to $\tilde{F}(M, Q)$ is very similar to the one of $\mathcal{F}_{\alpha}(M, Q)$ (see Fig. 1).

In the equal mass case, $\lambda=M$, we have an additional reparametrization symmetry, and the form factor is determined by Eq. (3) with the effective coupling $\bar{\alpha}=\alpha^{\prime}+$ $\alpha$ so that $\mathcal{F}(M, M, Q)=\mathcal{F}_{\bar{\alpha}}(M, Q)$. We can now write down the matching relation

$$
\mathcal{F}(M, M, Q)=C(M, Q) \tilde{F}(M, Q) \mathcal{F}_{\alpha^{\prime}}(M, Q),
$$

where the matching coefficient $C(M, Q)$ represents the effect of the power-suppressed terms neglected in Eq. (4). By combining the explicit results for $\mathcal{F}_{\alpha^{\prime}}(M, Q)$ and $\tilde{F}(M, Q)$ we find the two-loop matching coefficient

$$
\begin{aligned}
C(M, Q)= & 1+\frac{\alpha^{\prime} \alpha}{(4 \pi)^{2}}\left[\frac{59}{4}+\frac{70}{3} \pi^{2}+244 \zeta_{3}-\frac{113}{15} \pi^{4}\right. \\
& \left.-\frac{64}{3} \pi^{2} \ln ^{2} 2+\frac{64}{3} \ln ^{4} 2+512 \operatorname{Li}_{4}\left(\frac{1}{2}\right)\right] .
\end{aligned}
$$

Equation (8) does not contain logarithmic terms, and up to the $\mathrm{N}^{3} \mathrm{LL}$ accuracy the product $\tilde{F}(M, Q) \mathcal{F}_{\alpha^{\prime}}(\lambda, Q)$ continuously approaches $\mathcal{F}(M, M, Q)$ as $\lambda$ goes to $M$. Therefore, to get all the logarithms of the heavy gauge boson mass in two-loop approximation for the theory with mass gap, it is sufficient to divide the form factor $\mathcal{F}_{\bar{\alpha}}(M, Q)$ of the symmetric phase by the form factor $\mathcal{F}_{\alpha^{\prime}}(\lambda, Q)$ of the effective $\mathrm{U}_{\alpha^{\prime}}(1)$ theory taken at the symmetric point $\lambda=M$. Thus we have reduced the calculation in the theory with mass gap to the one in the symmetric theory with a single-mass parameter. Note that the absence of the linear-logarithmic term in Eq. (8) is an exceptional feature of the Abelian corrections. The general analysis of the evolution equation [9] shows the terms neglected in Eq. (4) to contribute starting from the $\mathrm{N}^{3} \mathrm{LL}$ approximation. This implies the absence of the second and higher powers of the logarithm in the matching coefficient of Eq. (7), irrespectively of the gauge group and the mass generation mechanism. Moreover, in the approximately equal mass case, $(M-$ $\lambda) / M \equiv \delta \ll 1$, one can compute the form factor as an expansion around the equal mass result. Up to $\mathrm{N}^{2} \mathrm{LL}$ accuracy only the leading term of Eq. (4) contributes and the expansion takes the form

$$
\begin{aligned}
\left.\mathcal{F}(\lambda, M, Q)\right|_{\lambda \rightarrow M}= & {\left[1-\delta \frac{\alpha^{\prime}}{\pi}\left(\mathcal{L}-\frac{3}{2}\right)\right] \mathcal{F}_{\tilde{\alpha}}(M, Q) } \\
& +\mathcal{O}\left(\delta^{2} \alpha^{\prime} \mathcal{L}, \delta \alpha^{\prime} \alpha \mathcal{L}\right) .
\end{aligned}
$$

Let us show how the above procedure applies to the calculation of two-loop electroweak corrections. To be specific, we consider a four-fermion neutral current process, which is of primary phenomenological importance, with light fermions. The four-fermion amplitude can be decomposed into (the square of) the form factor and a reduced amplitude [6,9]. The latter carries all the Lorentz and isospin indices and does not contain collinear logarithms in perturbative expansion. The logarithmic corrections to the reduced amplitude are obtained by solving a renormalization-group-like equation [18]. The corresponding two-loop anomalous dimensions can be extracted from the existing massless QCD calculations [19] (see $[9,20])$. Thus, the problem of the calculation of the two-loop electroweak logarithms in the four-fermion processes reduces to the analysis of the form factor.

In Ref. [9] by analyzing the hard evolution equation it has been found that the two-loop electroweak corrections up to the next-to-next-to-leading (quadratic) logarithms are not sensitive to the structure of the theory at the electroweak symmetry breaking scale. The prediction of Refs. [6,9] for the two-loop logarithmic corrections fully agrees with the available explicit results for the light fermion contribution [10] and the Abelian contribution obtained in this Letter. The only trace of the Higgs mechanism of the gauge boson mass generation in the $\mathrm{N}^{2} \mathrm{LL}$ approximation is the $Z-W$ boson mass splitting which can be systematically taken into account within an expansion around the equal mass approximation similar to Eq. (9). Thus, the calculation of the two-loop electroweak corrections up to the quadratic logarithms can be performed in two steps outlined above: (i) the corrections are evaluated using the fields of unbroken symmetry phase with all the gauge bosons of the same mass $M \approx$ $M_{Z, W}$ introduced by hand; (ii) the QED contribution with an auxiliary photon mass $M$ is factorized as in Eq. (7) leaving the pure electroweak logarithms. The separated virtual QED corrections accompanied by the real photon radiation in the limit of vanishing photon mass result in the universal infrared safe factor independent of $M_{Z, W}$.

By contrast, the $\mathrm{N}^{3} \mathrm{LL}$ approximation is sensitive to fine details of the gauge boson mass generation and the coefficient of the linear two-loop electroweak logarithm depends, e.g., on the Higgs boson mass. For the full calculation of this coefficient one has to use the true mass eigenstates of the standard model. Our result, Eqs. (2) and (6), is an example of such a calculation 
when applied to the two-loop diagrams with photon and $Z$ boson exchanges. We can, however, make a reasonable approximation which dramatically simplifies the analysis. Namely, consider a simplified model with a Higgs boson of zero hypercharge. Then the mixing is absent and the hypercharge gauge boson remains massless. The interference diagrams including the heavy $\mathrm{SU}_{L}(2)$ and the light hypercharge $\mathrm{U}(1)$ gauge bosons are identical with the ones of the purely Abelian model discussed in this Letter, where the above two-step procedure can be applied to get all the two-loop logarithms including the linear term. In the standard model the mixing of the gauge bosons results in a linear-logarithmic contribution, which is not accounted for within this procedure. It is, however, suppressed by a small factor $\sin ^{2} \theta_{W} \approx 0.2$, with $\theta_{W}$ being the Weinberg angle. Therefore, the above simplified model gives an estimate of the coefficient in front of the linear electroweak logarithm with $20 \%$ accuracy. From the numerical result of Fig. 1, which represents the typical structure of the two-loop corrections, we see that a $20 \%$ error in this coefficient leads to an uncertainty comparable to the nonlogarithmic contribution and is practically negligible. Thus we are able to get an accurate estimate of the two-loop correction, which is sufficient for practical applications to the future collider physics, by performing the calculations in the model without mass gap and mixing of the gauge bosons. The last ingredient necessary to complete the calculation of the dominant two-loop electroweak corrections is the generalization of Eq. (2) up to the linear-logarithmic term to the pure $\mathrm{SU}_{L}(2)$ gauge model with the Higgs mechanism of mass generation. Our result is sufficient to derive the total Abelian contribution due to photon and/or Z-boson exchanges to the two-loop linear logarithmic term in the four-fermion cross section. For example, for the $\nu_{e} \bar{\nu}_{e} \rightarrow$ $\nu_{\mu} \bar{\nu}_{\mu}$ cross section the correction has a simple analytical form, $\quad \delta \sigma=\left[-393 / 128+\pi^{2} / 32+3 \zeta_{3} / 8\right]\left[\alpha_{e w} /\right.$ $\left.\left(\cos ^{2} \theta_{W} \pi\right)\right]^{2} \ln \left(s / M_{Z}^{2}\right) \sigma_{\text {Born }}$.

To conclude, we have obtained the complete results for the two-loop corrections to the vector form factor in the Sudakov limit in Abelian theories with one massive gauge boson or with two gauge bosons of essentially different masses. Our approach solves the principal problems of the calculation of the dominant two-loop electroweak corrections to the neutral current four-fermion processes which are mandatory for the high-precision physics at the LHC and the next generation of linear colliders.

We thank S. Pozzorini for useful comments on the manuscript. J. H. K. acknowledges the hospitality of Kavli Institute for Theoretical Physics and partial support by the NSF under Grant No. PHY99-0794. The work of J. H. K and A. A. P. was supported in part by BMBF Grant No. 05HT4VKA/3 and Sonderforschungsbereich Transregio 9. The work of V. A. S. was supported in part by Volkswagen Foundation Contract No. I/77788, and DFG Grant No. Ha202/110-1.

[1] V.V. Sudakov, Zh. Eksp. Teor. Fiz. 30, 87 (1956) [Sov. Phys. JETP 3, 65 (1956)].

[2] R. Jackiw, Ann. Phys. (N.Y.) 48, 292 (1968); 51, 575 (1969).

[3] M. Kuroda, G. Moultaka, and D. Schildknecht, Nucl. Phys. B350, 25 (1991); G. Degrassi and A. Sirlin, Phys. Rev. D 46, 3104 (1992).

[4] M. Beccaria et al., Phys. Rev. D 58, 093014 (1998); P. Ciafaloni and D. Comelli, Phys. Lett. B 446, 278 (1999); J. H. Kühn and A. A. Penin, Karlsruhe Universität Report No. TTP/99-28; hep-ph/9906545.

[5] V.S. Fadin et al., Phys. Rev. D 61, 094002 (2000).

[6] J. H. Kühn, A. A. Penin, and V. A. Smirnov, Eur. Phys. J. C 17, 97 (2000); Nucl. Phys. B (Proc. Suppl.) 89, 94 (2000).

[7] M. Beccaria et al., Phys. Rev. D 61, 011301 (2000); 61, 073005 (2000); Phys. Rev. D 63, 053013 (2001); 64, 073008 (2001); A. Denner and S. Pozzorini, Eur. Phys. J. C 18, 461 (2001); 21, 63 (2001).

[8] M. Hori, H. Kawamura, and J. Kodaira, Phys. Lett. B 491, 275 (2000); W. Beenakker and A. Werthenbach, Nucl. Phys. B630, 3 (2002); A. Denner, M. Melles, and S. Pozzorini, Nucl. Phys. B662, 299 (2003); S. Pozzorini, Nucl. Phys. B692, 135 (2004).

[9] J. H. Kühn, S. Moch, A. A. Penin, and V. A. Smirnov, Nucl. Phys. B616, 286 (2001); B648, 455(E) (2002).

[10] B. Feucht, J. H. Kühn, and S. Moch, Phys. Lett. B 561, 111 (2003).

[11] J. Frenkel and J. C. Taylor, Nucl. Phys. B116, 185 (1976); D. Amati, R. Petronzio, and G. Veneziano, Nucl. Phys. B146, 29 (1978).

[12] See, e.g., G. Korchemsky, Phys. Lett. B 217, 330 (1989).

[13] M. Beneke and V. A. Smirnov, Nucl. Phys. B522, 321 (1998); V. A. Smirnov and E. R. Rakhmetov, Teor. Mat. Fiz. 120, 64 (1999); V. A. Smirnov, Phys. Lett. B 465, 226 (1999); , Applied Asymptotic Expansions in Momenta and Masses (Springer-Verlag, Heidelberg, 2001).

[14] G. Sterman, Phys. Rev. D 17, 2773 (1978); S. Libby and G. Sterman, Phys. Rev. D 18, 3252 (1978); A. H. Mueller, Phys. Rev. D 18, 3705 (1978); Phys. Rep. 73, 237 (1981).

[15] G. Kramer and B. Lampe, Z. Phys. C 34, 497 (1987); Z. Phys. C42, 504(E) (1989); T. Matsuura, S. C. van der Marck, and W. L. van Neerven, Nucl. Phys. B319, 570 (1989).

[16] A. H. Mueller, Phys. Rev. D 20, 2037 (1979); J. C. Collins, Phys. Rev. D 22, 1478 (1980); in Perturbative QCD, edited by A. H. Mueller (World Scientific, Singapore, 1989), p. 573; A. Sen, Phys. Rev. D 24, 3281 (1981).

[17] G. P. Korchemsky and A.V. Radyushkin, Nucl. Phys. B283, 342 (1987).

[18] A. Sen, Phys. Rev. D 28, 860 (1983).

[19] C. Anastasiou, E.W. N. Glover, C. Oleari, and M. E. Tejeda-Yeomans, Nucl. Phys. B601, 341 (2001); E.W. N. Glover, J. High Energy Phys. 04 (2004) 021.

[20] G. Sterman and M. E. Tejeda-Yeomans, Phys. Lett. B 552, 48 (2003). 